\title{
Observational study on
} safety and tolerability of duloxetine in the treatment of female stress urinary incontinence in German routine practice

Martin C. Michel, ${ }^{1}$ Anette Minarzyk, ${ }^{2}$ Inka Schwerdtner, ${ }^{2}$ Deborah Quail, ${ }^{3}$ Hans D. Methfessel ${ }^{4} \&$ Hans-Joachim Weber ${ }^{2}$

${ }^{1}$ Department of Pharmacology, Johannes Gutenberg University, Mainz, ${ }^{2}$ Medical Department, Lilly Germany GmbH, Bad Homburg, Germany ${ }^{3}$ European Statistics, Eli Lilly and Company, Windlesham, UK and ${ }^{4}$ Department of Gynecology, University Clinic Halle, Halle, Germany

\section{Correspondence}

Professor Martin C. Michel, Department of Pharmacology, Johannes Gutenberg University Mainz, Obere Zahlbacher Strasse 67, 55101 Mainz, Germany. Tel.: +4961327799000 Fax:+4961327299000 E-mail:

martin.michel@boehringer-ingelheim.com

Keywords

duloxetine, observational study, drug dosage, adverse events, suicidality

\section{Received}

9 February 2012

Accepted

17 July 2012

Accepted Article

Published Online

23 July 2012

\section{WHAT IS ALREADY KNOWN ABOUT THIS SUBJECT \\ - Duloxetine was safe in double-blind, randomized controlled trials but additional safety concerns have emerged for amine uptake inhibitors as a class, particularly with regard to suicidality. Clinically used duloxetine doses are often below the recommended doses.}

\section{WHAT THIS STUDY ADDS}

- Real life data confirm the tolerability and safety of duloxetine and in a population covering 3233 patient years no evidence for suicidality was seen. The prescribed duloxetine doses, which often are lower than the recommended ones, are associated with qualitatively similar but fewer adverse events than in controlled studies. Our parallel group design may be of value for observational studies in other therapeutic areas as well.

\section{AIMS}

To evaluate the safety and tolerability of duloxetine during routine clinical care in women with stress urinary incontinence (SUI) in Germany, and in particular, to identify previously unrecognized safety issues as uncommon adverse reactions, and the influence of confounding factors present in clinical practice on the safety profile of duloxetine.

\section{METHODS}

Office-based urologists, gynaecologists and primary care physicians were asked to document women newly started on treatment for moderate to severe symptoms of SUI. Six thousand eight hundred and fifty-four patients from urologist/gynaecologist practices and 5879 primary care patients were assessed. In a two-armed, observational study with parallel 12 week (urologists and gynaecologists) or 24 week (primary care physicians) design, patients were treated with duloxetine or other conservative treatment. The main outcome measure was the occurrence of adverse events (AEs).

\section{RESULTS}

Baseline characteristics differed slightly between patient groups and studies. Duloxetine doses in most patients were lower than recommended. Overall, AE frequency with duloxetine was lower than in controlled studies $(15.9 \%(95 \% \mathrm{Cl}$ $14.9,16.9)$ and $9.1 \%(95 \% \mathrm{Cl} 8.2,10.0)$ in the 12 and 24 week treatment groups, respectively), but exhibited a similar qualitative spectrum. In the logistic regression models, the following factors were associated with greater $A E$ risk: investigator specialization (gynaecologist vs. urologist and primary care physician), initial duloxetine dose ( $80 \mathrm{vs} .20 \mathrm{mg} \mathrm{day}^{-1}$ ) and use of any concomitant medication. Within the 24 week study, a positive screen for depressive disorder was surprisingly common, but no case of attempted suicide was reported in either study.

\section{CONCLUSIONS}

Our results from German clinical practice show that women with SUI were often treated with duloxetine doses lower than recommended. This was associated with a low incidence of AEs. Suicide attempts were not reported. 


\section{Introduction}

Stress urinary incontinence (SUI) is defined as the involuntary leakage of urine on effort, exertion, sneezing or coughing [1]. SUI accounts for approximately half of all urinary incontinence cases experienced by adult women [2-4] and is associated with substantial impairment of quality of life [5-7] and a considerable financial burden to the patients and the health care systems [1]. Historically, the treatment of SUI consisted of conservative measures (e.g. pessaries, pads, pelvic floor muscle training) and, in severe cases, surgery. Local treatment with oestrogens is often being applied [8] despite a lack of regulatory approval.

Duloxetine is a mixed serotonin-norepinephrine re-uptake inhibitor (SNRI) which is registered in many countries around the globe for the treatment of depression and pain associated with diabetic peripheral neuropathy. Moreover, it is the first medication approved for the treatment of moderate to severe female SUI in Europe. Experimental studies in animals $[9,10]$ and healthy female volunteers [11] suggest that duloxetine enhances the excitability of the pudendal motor neurons and urethral sphincter contractility, and suppresses bladder activity. Its pharmacological properties have been reviewed comprehensively [12]. The clinical efficacy of duloxetine in the treatment of SUI has been documented in a range of placebo-controlled, randomized, doubleblind studies [13-17]. Additional studies have demonstrated its efficacy in the treatment of mixed incontinence [18] or in combination with pelvic floor muscle training [19].

The adverse events (AEs) seen in the above controlled clinical trials in women with SUI and in a pooled analysis thereof [20] were mainly nausea and dizziness. A recent meta-analysis of AEs in placebo-controlled studies across all indications reported a qualitatively similar safety profile as in the SUI studies, although with slightly different incidences dependent on indication [21]. Rare but potentially important AEs originally reported for antidepressants from the selective serotonin re-uptake inhibitor (SSRI) class are suicidal ideations and suicide attempts [22, 23]. Whether such risk applies similarly to patients with depressive disorders as to those with other psychiatric conditions or with non-psychiatric conditions such as SUI is not well understood but one recent analysis of almost 100000 patients receiving antidepressants or placebo found that suicidal behaviour or ideation was extremely rare when used in non-psychiatric indications [23]. Label warnings for both SSRIs and SNRIs reflect that this is an important identified risk for all classes of antidepressants, and for safety considerations apply this warning to all uses of these drugs. This situation merits evaluation of suicidality in SUI for SNRI including duloxetine as the available controlled studies have been under-powered to detect such a risk in the SUI patient population and/or have excluded the most vulnerable SUI patients [13-19].
Based upon the controlled studies in SUI patients, duloxetine treatment of SUI is generally considered to be safe and well tolerated. However, the safety and tolerability profile of duloxetine observed in controlled studies may not be fully representative of that in routine clinical practice for several reasons. Firstly, controlled studies typically are of a limited size and hence have a limited statistical power to detect infrequent AEs. Secondly, controlled studies typically have long lists of specific inclusion and exclusion criteria which may bias against those patients who are most vulnerable. Thirdly, most SUI patients in the controlled clinical studies had been recruited by urologists (UROs) or gynaecologists (GYNs), whereas many SUI patients in routine practice are being treated by primary care physicians $(\mathrm{PCP})$. Depending on the health care system in a given country, the SUI patients receiving specialist care vs. non-specialist care may exhibit different characteristics with regard to disease severity, disease duration and/or comorbidity.

Against this background, the DUROSA ('Duloxetine Routine Safety') study was started in February 2005, shortly after the approval of duloxetine for the treatment of female SUI in Germany. Its study design and protocol amendments largely reflect specific requests by the European Medicines Agency. The primary objective was to evaluate the safety and tolerability of duloxetine during routine clinical care in women with SUI in Germany, and in particular, to identify previously unrecognized safety issues such as uncommon adverse reactions, and the influence of confounding factors present in clinical practice on the safety profile of duloxetine. Additional objectives of this study were the confirmation of the established safety profile of duloxetine and the characterization of the female SUI patient population in an everyday clinical practice setting in Germany. Interim data on dosing decisions in this study have been published previously [24].

\section{Methods}

\section{Setting}

DUROSA was set up as a two-armed, open-label, prospective, observational, post-authorization safety study. Mostly office-based UROs $(n=380)$, GYNs $(n=236)$ and PCPs $(n=1073)$ throughout Germany were asked to document systematically women who were initiated to a new conservative treatment for SUI, either with duloxetine (DULOX) or some reference therapy (OTHER). They were also asked to document one patient with OTHER therapy per every two DULOX patients, on respective case report forms.

Recruitment started in February 2005 and the last patient completed the study in July 2008. The planned observation period was 12 weeks, but based upon a request by the European Medicines Agency was extended to 24 weeks for participants recruited by PCPs as an addendum to the original study protocol, together with a modi- 
fied set of data capturing forms. Therefore, we largely report the results as two parallel studies, one 12 week study by UROs and GYNs with 6854 patients (DULOX-12, OTHER-12) and one 24 week study by PCPs with 5879 patients (DULOX-24, OTHER-24). In deviation from the protocol, the 12 week study also documented $44(0.6 \%)$ patients treated by PCPs, and in 573 (8.4\%) patient information on physician specialization was missing. In the 24 week study 57 (1.0\%) patients were treated by specialists (UROs and/or GYNs) and 442 (7.5\%) lacked data on physician specialization.

\section{Sample size consideration}

The primary objective of this study was, in particular, to establish the frequency of potential uncommon adverse events (i.e. those with incidences between $0.1 \%$ and $1 \%$ ) in SUI patients treated with duloxetine. It was therefore planned to document approximately 16000 DULOX and 8000 OTHER patients. Thus, an uncommon adverse drug reaction with an incidence of $1 \%$ could be detected with a $95 \%$ confidence interval $(\mathrm{Cl})$ of $\pm 0.16 \%$. However, when it became clear from the actual recruitment rates after the start of the study that these numbers of patients would not be reached within the planned time, the inclusion target was reduced to 8000 and 4000 patients, respectively. This smaller number still allowed the detection of an $\mathrm{AE}$ with an incidence of $1 \%$ with a precision of $\pm 0.23 \%$, which was considered adequately precise still to meet the study objectives.

\section{Treatment}

Based upon the package insert, the recommended target duloxetine dose was $80 \mathrm{mg} \mathrm{day}^{-1}$, either from the start or following an up-titration period. All types of newly started conservative treatment were admissible in the OTHER group including pelvic floor muscle training (PMFT), pessaries and hormonal treatment. The choice of treatment for the individual patient was entirely at the discretion of her physician. Adjunct and combination therapy was acceptable in both treatment groups. Therapy could be changed during the course of the observation without discontinuing the patient from the study (except when the patient switched to surgical treatment).

\section{Patient selection and documentation}

There were no specific inclusion criteria other than the presence of moderate to severe SUI symptoms in women with a minimum age of 18 years, and no exclusion criteria other than planned SUI surgery during the observation period and the recommendations from the applicable Summary of Product Characteristics. Mixed forms of urinary incontinence were acceptable. The participating physicians were asked to record systematically their observations in patients receiving duloxetine or other conservative treatment based on their medical judgment. In line with the observational character of our study, there was no specific training of participating physicians in the detection of AEs. Rather AEs were captured by an open question on the case record form ('Have there been AEs? If so, which?'; see below).

Documentation started with the patient's initiation on a new SUI treatment (baseline). Further documentation of SUI treatment and of treatment outcomes including AEs was scheduled 4 and 12 weeks thereafter and additionally after 24 weeks in the PCP study. At baseline, the following data were collected: age, height, weight, number and type of deliveries, duration of SUI symptoms, incontinence episode frequency per week (IEF), number of pads used per week, previous treatment of SUI, menopausal status and the physician's specialization. Relevant concomitant diseases and concomitant medications were documented to allow for further assessment of possible causality of treatment emergent AEs. AEs were solicited by general questioning at all post-baseline visits, including start and stop dates as well as the investigator's assessment of causality, and coded according to the Medical Dictionary for Regulatory Activities (MedDRA). Discontinuation rates, including reasons for discontinuation, were reported separately for patients following the 12 and 24 week studies. The initial daily dose of duloxetine was documented at baseline. Duloxetine dosage was reassessed at every following visit, documenting any change.

Apart from the additional follow-up visit at week 24, PCPs did a baseline screen on depressive core symptoms, suicidality and self-harm, using the Dep-2 depression score $[25,26]$, which consists of two questions rating depressive mood and anhedonia on a five step scale (no, never $=0$ to every day $=4$ ). Patients with a total score of $\geq 5$ were rated 'most likely cases of depressive disorder'. Suicidal ideation, thoughts of self harm and history of suicide attempts were assessed with three additional questions ('Did you think about suicide or self harm during the last 2 weeks?' - 'Did you ever think about suicide or self harm?' - 'Did you ever attempt suicide or harm yourself'). Of note Dep-2 is a screening tool only and not a formal tool to diagnose depression.

\section{Statistical analysis}

Unless otherwise specified, DULOX and OTHER patients were analyzed separately for the 12 and 24 week study. The analysis was largely descriptive. For quantitative variables (e.g. age) arithmetic means, medians, standard deviations, minimum and maximum values, quartiles as well as the respective available sample size and the number of missing values per category were calculated. Qualitative variables were described using absolute and relative frequencies (adjusted relative frequencies where appropriate).

Two sided $95 \% \mathrm{Cls}$ were calculated for the rates of AEs and serious AEs (SAEs). The association of relevant confounding factors with occurrence of any $A E$, gastrointestinal AEs and nausea in the DULOX cohort, was assessed 
through step-wise logistic regression models. The following models were calculated: (1) One pooled analysis, comprising the patients from DULOX-12 and DULOX-24, including all AEs irrespective of the observation period (DULOX-AII), (2) two separate models for all DULOX patients (DULOX-All 12) and all OTHER (OTHER-All-12) comprising the pooled AEs of the respective treatment groups occurring during the first 12 weeks and (3) one model for DULOX-24 only. Independent variables included into the models comprised investigator specialization, initial dose of duloxetine, overactive bladder medication, antidepressant co-medication, other concomitant medication, other comorbidities (excluding depression), BMI, age and depressive symptoms at baseline according to baseline screen (for DULOX-24 model only).

The study was conducted according to the ethical principles of the Declaration of Helsinki and approved by the ethics committee of the Georg-August University Göttingen, Germany. The patients provided written consent to the collection and release of anonymous data regarding treatment and its outcomes. The study was compliant with regulatory guidelines for observational studies in Germany.

\section{Results}

\section{Patient characteristics}

In both studies, DULOX patients were slightly older and slightly more likely to be post-menopausal than patients from the respective OTHER group (Table 1). The duration of
SUI and its severity were higher in DULOX-12 than in OTHER-12 group (47.0 vs. 39.5 months, IEF 16.3 vs. 12.9), but similar in both groups of the 24 week study (43.3 vs. 44.0 months, IEF 10.9 vs. 9.9, Table 1). Moreover, DULOX-12 patients were more likely to have ongoing other conservative treatments at baseline, largely pelvic floor muscle training, than OTHER-12 patients (Table 1). In line with their greater age, DULOX patients in both studies reported more comorbidities than the respective OTHER patients. Hypertension, lipid metabolism disorders and diabetes were the most frequent comorbidities in both treatment groups (Table 2).The reported use of comedications was similar in DULOX and OTHER patients, with antihypertensives being used most frequently. Compared with DULOX-12 and OTHER-12, DULOX-24 and OTHER-24 comprised patients of slightly higher age and less severe SUI symptoms with more comorbidities and more comedications than the corresponding 12 week groups (Table 2). Of note, 17 patients reported concomitant use of duloxetine prescribed in its antidepressant indication under a different brand name (1 DULOX-12, 11 DULOX-24, 1 OTHER-12, 4 OTHER-24). Concomitant use of overactive bladder medications, reflecting that the study protocol allowed inclusion of patients with mixed incontinence, was less frequent in DULOX patients (4.9\% and $1.8 \%$ in the 12 and 24 week studies, respectively) than in OTHER patients $(16.0 \%$ and $14.7 \%$, respectively, see Table 2). Among those, trospium, oxybutynin and tolterodine were reported most frequently (all others $<1 \%$ in each cohort).

The depression screening in DULOX-24 and OTHER-24 yielded scores of 0 (depression unlikely) in $33.8 \%$ and

\section{Table 1}

Baseline characteristics of cohorts. Data are number of patients (\% of cohort in parentheses), means \pm SD or $\%$ of cohort. Multiple nominations were possible for ongoing treatments at baseline

\begin{tabular}{|c|c|c|c|c|}
\hline & \multicolumn{2}{|c|}{12 week study } & \multicolumn{2}{|c|}{24 week study } \\
\hline & DULOX-12 & OTHER-12 & DULOX-24 & OTHER-24 \\
\hline Number of patients & 4913 & 1941 & 4010 & 1869 \\
\hline Post-menopausal & 78.5 & 72.3 & 84.0 & 81.5 \\
\hline BMI $\left(\mathrm{kg} \mathrm{m}^{-2}\right)$ & $26.7 \pm 4.2$ & $26.1 \pm 4.0$ & $27.6 \pm 4.3$ & $27.1 \pm 4.3$ \\
\hline Number of pregnancies & $2.2 \pm 1.4$ & $2.1 \pm 1.4$ & $2.1 \pm 1.4$ & $2.0 \pm 1.4$ \\
\hline Number of child deliveries & $2.0 \pm 1.2$ & $1.9 \pm 1.2$ & $2.0 \pm 1.3$ & $1.9 \pm 1.3$ \\
\hline IEF (episodes per week) & $16.3 \pm 13.6$ & $12.5 \pm 10.8$ & $10.9 \pm 10.2$ & $9.9 \pm 9.2$ \\
\hline Pads used per week & $17.7 \pm 11.4$ & $14.8 \pm 9.9$ & $15.6 \pm 11.1$ & $14.8 \pm 11.6$ \\
\hline Any previous SUI treatment & 56.9 & 37.9 & 45.1 & 42.9 \\
\hline Previous SUI surgery & 10.6 & 9.0 & 2.3 & 2.2 \\
\hline \multicolumn{5}{|c|}{ Additional SUI treatment (baseline) } \\
\hline Any & $2700(55.0)$ & $868(44.7)$ & $1609(40.1)$ & $756(40.4)$ \\
\hline Other & $119(2.4)$ & $95(4.9)$ & $54(1.3)$ & $42(2.2)$ \\
\hline
\end{tabular}

BMI, body mass index; IEF, incontinence episode frequency; PMFT, pelvic floor muscle training 


\section{Table 2}

Comorbidities and comedications at baseline by cohort. Data are number of patients with $\%$ of cohort in parentheses. Only items occurring in at least $1 \%$ of patients in at least one cohort are presented. Multiple nominations were possible

\begin{tabular}{|c|c|c|c|c|}
\hline \multirow[b]{2}{*}{ Comorbidities } & \multicolumn{2}{|c|}{12 week study } & \multicolumn{2}{|c|}{24 week study } \\
\hline & $\begin{array}{l}\text { DULOX-12 } \\
n(\%)\end{array}$ & $\begin{array}{l}\text { OTHER-12 } \\
n(\%)\end{array}$ & $\begin{array}{l}\text { DULOX-24 } \\
n(\%)\end{array}$ & $\begin{array}{l}\text { OTHER-24 } \\
n(\%)\end{array}$ \\
\hline Any comorbidity & $2531(51.5)$ & $922(47.5)$ & $2892(72.1)$ & $1266(67.7)$ \\
\hline Hypertension & $1825(37.2)$ & $672(34.6)$ & $2175(54.2)$ & $911(48.7)$ \\
\hline Lipid metabolism disorder & $659(13.4)$ & $253(13.0)$ & $1419(35.4)$ & 646 (34.6) \\
\hline Diabetes/pre-diabetes & $792(16.1)$ & $277(14.3)$ & $905(22.6)$ & $413(22.1)$ \\
\hline Heart disease & $352(7.2)$ & $110(5.7)$ & $517(12.9)$ & $197(10.5)$ \\
\hline Depression & $185(3.8)$ & $105(5.4)$ & $681(17.0)$ & $300(16.1)$ \\
\hline Bronchopulmonary disease & $216(4.4)$ & $77(4.0)$ & $258(6.4)$ & $124(6.6)$ \\
\hline Neurogenic urinary hesistancy & $55(1.1)$ & $21(1.1)$ & $44(1.1)$ & $21(1.1)$ \\
\hline Coagulation disorder & $51(1.0)$ & $20(1.0)$ & $45(1.1)$ & $16(0.9)$ \\
\hline Liver diseases & $24(0.5)$ & $10(0.5)$ & $63(1.6)$ & $25(1.3)$ \\
\hline \multicolumn{5}{|l|}{ Comedications } \\
\hline Any specification & $2333(47.5)$ & $956(49.3)$ & $2463(61.4)$ & $1142(61.1)$ \\
\hline Antihypertensives & $1720(35.0)$ & $609(31.4)$ & $2119(52.8)$ & $890(47.6)$ \\
\hline Hormones & $465(9.5)$ & $137(7.1)$ & $207(5.2)$ & $78(4.2)$ \\
\hline Anticoagulants & $258(5.3)$ & $97(5.0)$ & $312(7.8)$ & $114(6.1)$ \\
\hline Antidepressants & $120(2.4)$ & $87(4.5)$ & $433(10.8)$ & $209(11.2)$ \\
\hline Duloxetine as antidepressant & $1(<0.1)$ & $1(0.1)$ & $11(0.3)$ & $4(0.2)$ \\
\hline Theophylline & $70(1.4)$ & $32(1.7)$ & $132(3.3)$ & $46(2.5)$ \\
\hline Sedatives & $53(1.1)$ & $28(1.4)$ & $217(5.4)$ & $105(5.6)$ \\
\hline Overactive bladder medication & $239(4.9)$ & $310(16.0)$ & $73(1.8)$ & $274(14.7)$ \\
\hline Trospium chloride & $74(1.5)$ & $104(5.4)$ & $25(0.6)$ & $66(3.5)$ \\
\hline Oxybutynin & $61(1.2)$ & $77(4.0)$ & $21(0.5)$ & $116(6.2)$ \\
\hline Tolterodine & $56(1.1)$ & $62(3.2)$ & $10(0.3)$ & $52(2.8)$ \\
\hline Solifenacin & $27(0.6)$ & $48(2.5)$ & $7(0.2)$ & $43(2.3)$ \\
\hline Other anticholinergic & $19(0.4)$ & $22(1.1)$ & $1(<0.1)$ & $11(0.6)$ \\
\hline Darifenacin & $18(0.4)$ & $37(2.0)$ & $2(0.1)$ & $17(0.9)$ \\
\hline Other & $16(0.3)$ & $8(0.4)$ & $16(0.4)$ & $16(0.9)$ \\
\hline
\end{tabular}

$38.3 \%$, of 1-2 (possible symptoms of depressed mood) in $24.0 \%$ and $23.5 \%$, of 3-4 (probable case of depressive disorder) in $26.6 \%$ and $25.1 \%$ and of $\geq 5$ (most likely case of depressive disorder) in $15.6 \%$ and $13.2 \%$, respectively. Accordingly, thoughts of self-harm were reported by $4.4 \%$ and $5.0 \%$, suicidal thinking by $9.8 \%$ and $8.5 \%$ and a history of suicide attempts by $1.7 \%$ and $1.6 \%$, respectively.

Patient flow and details on patient disposition are shown in Figure 1. Early discontinuation occurred in 542 (11.0\%) DULOX-12 and 230 (5.7\%) DULOX-24 patients and in 97 (5.0\%) OTHER-12 and 79 (4.2\%) OTHER-24 patients. The most frequent reasons for discontinuation were AEs and 'other reason' in DULOX-12 and DULOX-24 patients. In the OTHER groups 'surgical procedure', 'other reason' and 'SUI therapy stopped' were reported most frequently (Table 4), possibly the 'other reason' group also included patients with insufficient treatment efficacy.

\section{Initial treatment}

The initial dose in DULOX patients in the 12 and 24 week studies was $20 \mathrm{mg} \mathrm{day}^{-1}$ (26.9\% and $30.4 \%$, respectively), $40 \mathrm{mg} \mathrm{day}^{-1} \quad(54.7 \%$ and $48.5 \%$, respectively $)$ and
$80 \mathrm{mg} \mathrm{day}^{-1}$ (17.9\% and $20.4 \%$, respectively). While doses were decreased in a few patients during the course of the study, about half of all patients who started on doses lower than $80 \mathrm{mg} \mathrm{day}^{-1}$ were up-titrated within the first 4 weeks. At study end the prescribed doses in the 12 and 24 week studies were $20 \mathrm{mg}$ day $^{-1}$ in $16.1 \%$ and $22.3 \%, 40 \mathrm{mg}$ day $^{-1}$ in $43.1 \%$ and $36.1 \%$ and $80 \mathrm{mg} \mathrm{day}^{-1}$ in $39.2 \%$ and $40.5 \%$, respectively. Treatments newly initiated at baseline in the 12 and 24 week OTHER groups were pelvic floor muscle training (65.8\% and $47.7 \%)$, local hormone treatment (12.0\% and $11.0 \%)$, homeopathy (1.4\% and $16.9 \%)$, pessaries $(6.9 \%$ and $5.1 \%)$ and other treatments $(13.8 \%$ and 19.4\%; multiple nominations possible).

\section{Safety and tolerability}

Treatment-emerging AEs were reported in $15.9 \%(95 \% \mathrm{Cl}$ 14.9, 16.9\%) DULOX-12 and 3.1\% (95\% Cl 2.4, 4.0\%) OTHER-12 patients (Table 3). Corresponding numbers for DULOX-24 and OTHER-24 were 9.1\% (95\% Cl 8.2, 10.0\%) and $5.7 \%$ (95\% Cl 4.7,6.9\%). SAEs were reported in $0.1 \%$ (95\% Cl 0.0, 0.3\%) DULOX-12 and <0.1\% (95\% Cl 0.0, 0.3\%) OTHER-12 patients and in $0.5 \%(95 \% \mathrm{Cl} 0.3,0.7 \%)$ 


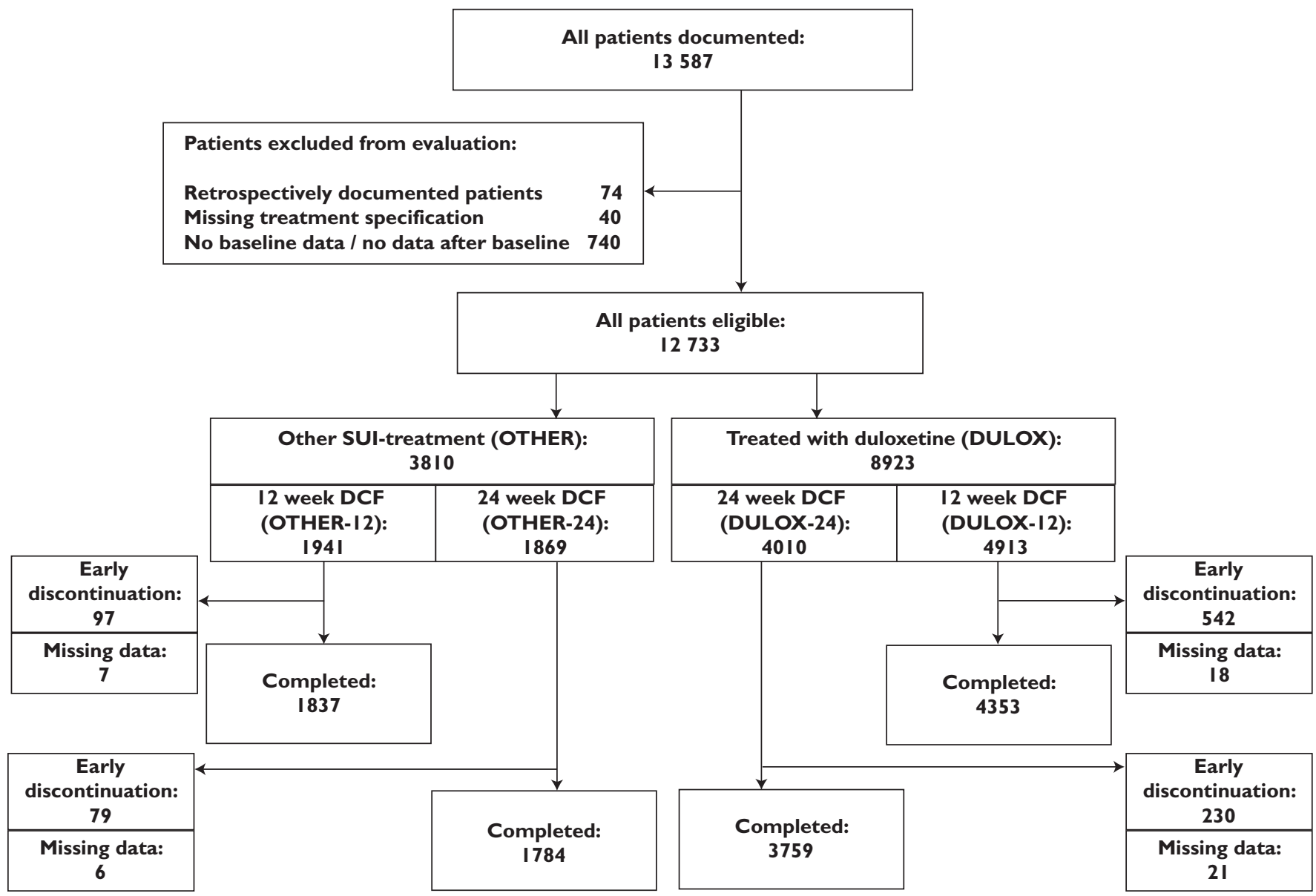

\section{Figure 1}

Patient flow

\section{Table 3}

Adverse events (AEs). Data are presented as absolute number per group with \% values in parentheses (relative to cohort size). Specific AEs are listed if they occurred in at least $0.5 \%$ of at least one group; multiple nominations possible

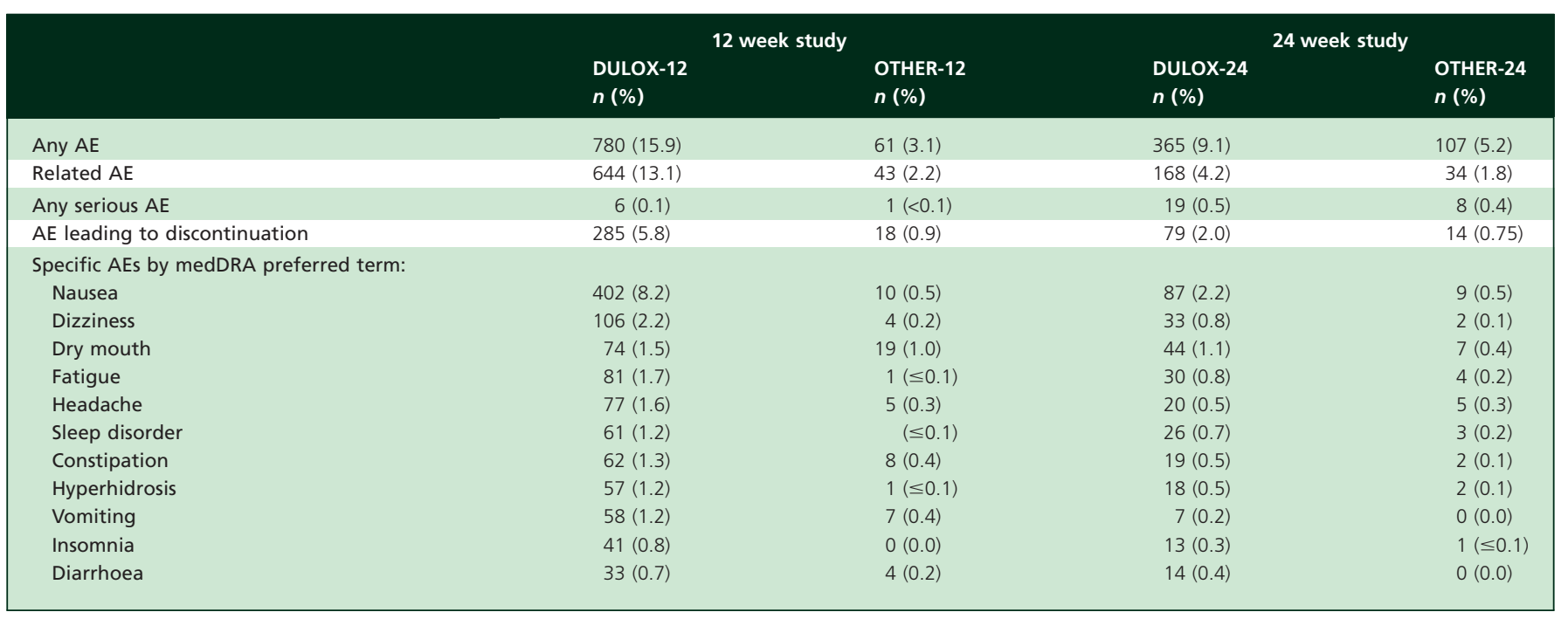




\section{Table 4}

Reasons for discontinuation. Data are presented as absolute number per group with \% values in parentheses (relative to cohort for all specific reasons; multiple nominations possible)

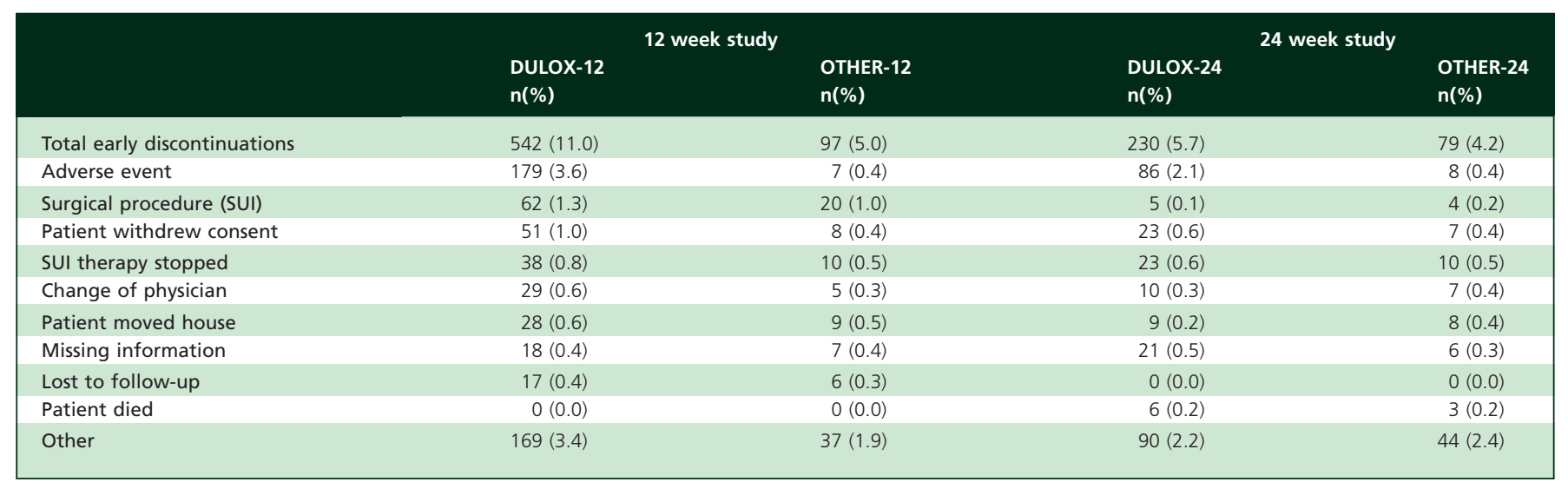

DULOX-24 and $0.4 \%$ (95\% Cl 0.2, 0.8\%) OTHER-24 patients. AEs leading to discontinuation were reported in 5.8\% (95\% $\mathrm{Cl} 5.2,6.5 \%)$ DULOX-12 and $0.9 \%(95 \% \mathrm{Cl} 0.6,1.5 \%)$ OTHER-12 patients and in $2.0 \%(95 \% \mathrm{Cl} 1.6,2.5 \%)$ DULOX-24 and $0.8 \%$ (95\% Cl 0.4, 1.3\%) OTHER-24 patients (Table 3). In total, 12 treatment-emerging AEs with a fatal outcome were reported, occurring in one DULOX-12 patient (one 'natural death'), and eight DULOX-24 patients (one perforated sigma diverticulum, one acute cardiac failure, one decompensated heart insufficiency, pneumonia and tachyarrhythmia, one myocardial infarction, one intestinal infarction, one road traffic accident, one rectal cancer, infection, sepsis and multi-organ failure, one endometrial cancer) and three OTHER-24 patients (one 'cardiovascular disease', one cardiac arrest, one apoplectic insult). There was no case of fatal suicide and no fatality judged to be treatment related.

The most frequent types of AEs in both DULOX groups were nausea, dizziness, dry mouth, fatigue, headache and sleep disorder (Table 3). The most frequent types of AEs in both OTHER groups were dry mouth, nausea, headache and constipation (Table 3). Suicide attempts were not reported by any patient.

To identify factors associated with treatment-emerging AEs, we applied stepwise regression models (see Methods). The stepwise logistic regression model for DULOX-All 12 showed that the following factors were significantly associated with the occurrence of any type of AE: investigator specialization (patients from GYN practices had a higher risk than patients from PCPs or UROs), initial duloxetine dose (patients initiated with $80 \mathrm{mg}$ vs. $20 \mathrm{mg}$ showed higher $\mathrm{AE}$ risk), intake of concomitant overactive bladder medication, antidepressants or any other concomitant medication (Table 5). The DULOX-24 model showed a similar pattern with greater age being an additional significant factor, whereas a 'most likely or probable' result from the baseline depression screen was associated with a lower risk of AEs (Table 5). Significant factors in OTHER-All 12 were intake of overactive bladder medication or any other concomitant medication (Table 5). Regression models for gastrointestinal AEs or specifically for nausea yielded comparable results (data not shown).

\section{Discussion}

\section{Critique of methods}

Controlled clinical studies and observational studies should not be viewed as mutually exclusive but rather as exploring different types of questions and having distinct advantages and limitations. Controlled studies compare one treatment with a reference treatment (often placebo) under specifically defined conditions generating high internal validity, specifically when randomized and, where possible, blinded. However, these specific inclusion and exclusion criteria also limit extrapolation to the affected patient population at large and hence external validity. Related at least partly to the associated costs, controlled studies typically also have a limited sample size, and in most cases are powered to detect differences in efficacy but often under-powered to detect differences in tolerability, particularly with regard to less frequent AEs. In contrast, observational studies typically lack a control group and hence have limited internal validity. However, due to very limited inclusion and exclusion criteria they are more reflective of the afflicted patient population and hence have higher external validity. They also often include much greater patient numbers allowing for a greater probability of detecting less frequent AEs. Such greater patient numbers also allow the application of multiple regression models to explore relationships between treatment outcomes and various potentially explanatory variables. The present study uses a novel design in the field of observational studies by including a reference group receiving 


\section{Table 5}

Results from stepwise logistic regression models to identify factors significantly associated with treatment-emerging AEs. Shown are results for the pooled DULOX groups during the entire study $(12+24$ weeks; DULOX-ALL whole study), the pooled DULOX groups during the first 12 weeks (DULOX-ALL-12), the DULOX group of the 24 week study (DULOX 24) and the pooled OTHER group during the first 12 weeks (OTHER-ALL-12). Shown are $P$ values and corresponding odds ratio, with a value $<1$ or $>1$ indicating a better or worse risk as compared with the respective reference variable. NS: not significant

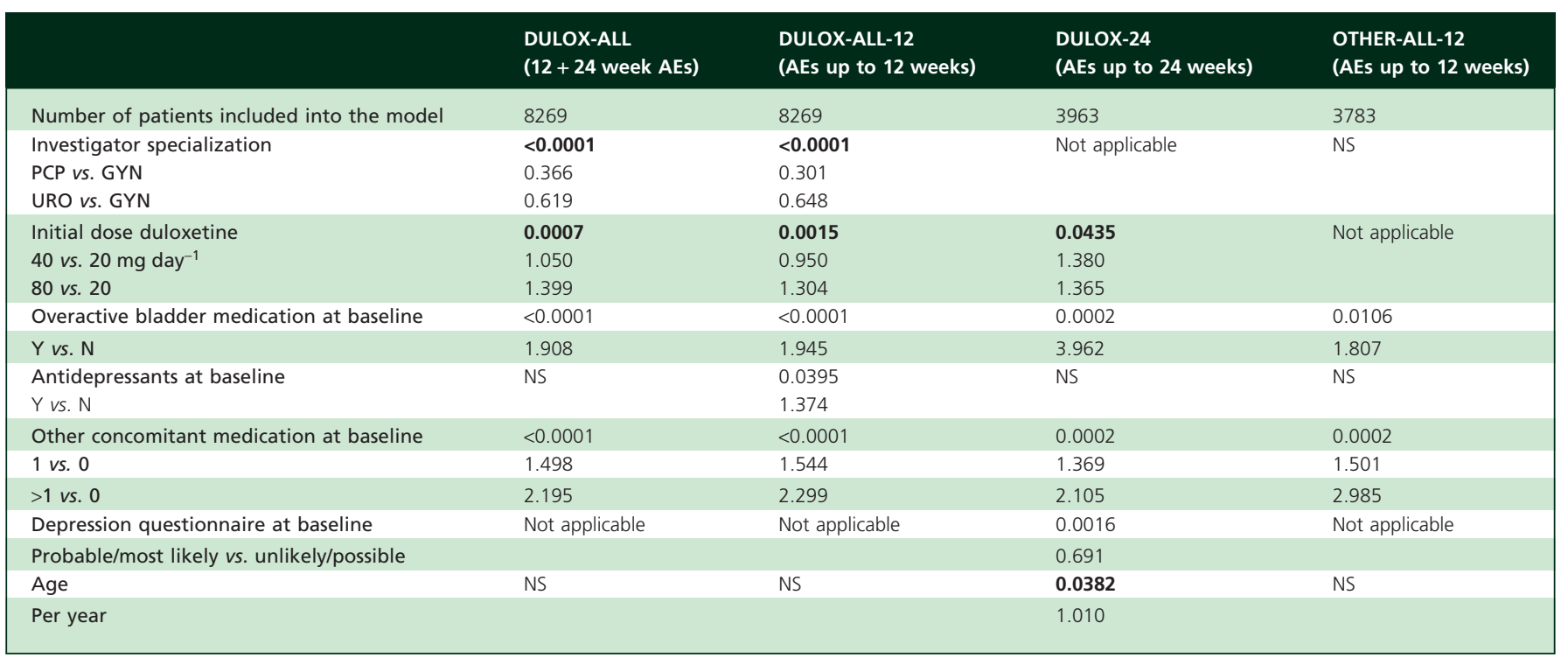

other conservative treatments. While this does not create the same internal validity as in a randomized controlled trial, this perhaps is the closest to a comparator group which can be achieved without violating the principles of an observational study. Indeed, the DULOX groups recruited by both specialists and PCPs were slightly older and had more severe SUI compared with the OTHER group. The occurrence of AEs in the treatment of urinary incontinence may be age-dependent $[27,28]$ and, perhaps even more importantly, the treatments in the OTHER group, such as pelvic floor muscle training, largely represent approaches typically not associated with AEs by patients. Therefore, the OTHER group may not be a good control for overall AEs and accordingly we did not perform statistical comparisons for this parameter between the two groups. On the other hand, analyses of suicidal behaviour and ideation in patients receiving duloxetine or escitalopram have found that their incidence varies more between studies than between active treatment and placebo groups [29]. This would have made it very difficult to assess the value of the data for suicidal behaviour or ideation in the present study if we had not had included the OTHER group as an internal reference.

There are additional differences between the present observational and most previously reported randomized duloxetine studies in SUI patients. Firstly, except for one phase IV study of SUI [30] published after initiation of the present study, all randomized duloxetine studies had not involved any dose-escalation but had started all patients on the target dose. In line with the observational character of our study, we had not specified duloxetine doses to be used. Concomitant with the start of our study the results of the randomized dose-escalation study became known and apparently were taken up rapidly among German physicians but without routinely escalating to the recommended target dose. Secondly, also in line with the observational character of the present study, there were no specific recommendations to participating physicians as to how to record treatment-emerging AEs other than an open question in the case-record forms. Thirdly, the present study did not specify inclusion and exclusion criteria other than those defined in the Summary of Product Characteristics, and we consider this to be a strength of the present study with regard to its external validity. Finally, and perhaps most importantly with regard to AE reporting, patients receiving medication as part of routine care generally tend to report fewer AEs than those in randomized trials, probably because being a patient receiving routine care creates a different level of side effect awareness from being a participant in a study exploring an investigational agent. Accordingly, low AE incidences as compared with the earlier randomized studies have routinely been observed in observational studies on other incontinence medications [27, 28, 31, 32]. All of these factors may have contributed to a low reported incidence of $A E s$ in the present study.

Reflecting real-life conditions, our study has recruited SUI patients treated by specialists (UROs, GYNs) and by PCPs. Indeed, our findings demonstrate that specialists and PCPs treat SUI patients with different baseline characteris- 
tics. While UROs/GYNs tended to prescribe duloxetine to more severely affected patients than PCPs, PCPs recruited somewhat older patients and reported higher rates of comorbidity and concomitant medications. Based upon a request by regulatory authorities, the study protocol for PCPs differed with regard to the duration of the planned observation period and also specific baseline assessments of depression and suicidality. In this context it should also be mentioned that the Dep-2 used by us is a screening tool and does not allow a formal diagnosis of depression. Moreover, this screening tool has not been validated to monitor changes over time and hence was applied to baseline data only.

Similar to overactive bladder medications [33], duloxetine was largely prescribed to post-menopausal, slightly overweight women with about two previous pregnancies. Of note an average of 11-16 incontinence episodes week ${ }^{-1}$ is less than expected based upon the registered indication of duloxetine for medium to severe SUI. As reported earlier [24], all three physician groups tended to start SUI patients on rather low duloxetine doses, often lower than the lowest dose recommended in the Summary of Product Characteristics. This may partly reflect findings that starting with a low dose followed by up-titration may optimize the tolerability of duloxetine [30], but it should be noted that the majority of patients in the present study never reached the recommended therapeutic dose of $80 \mathrm{mg} \mathrm{day}^{-1}$ that was used in the controlled trials. While we have no hard efficacy data from the present safety study, it is possible based on the published dose-response relationship for duloxetine efficacy in SUI patients [13], that many patients in the present study did not experience the full therapeutic benefit of the drug.

\section{Safety and tolerability findings}

The overall pattern of AEs observed in the present observational study is qualitatively similar to what previously has been reported from controlled studies with duloxetine in women with SUI [13-19]. However, the absolute incidence of AEs was considerably lower in the present observational than in previously reported controlled studies, e.g. for nausea $8.2 \%$ and $2.2 \%$ in the present specialist and PCP cohorts as compared with $23.2 \%$ in the combined phase II/III studies [20]. While this can partly be attributed to the general observation that observational studies report lower $\mathrm{AE}$ rates than controlled studies, two other reasons should be noted. Firstly, apparently most physicians prescribing duloxetine applied dose-escalation upon initiation of treatment, which has been shown to reduce the frequency of AEs [30]. Secondly, many patients had final duloxetine doses below the recommended $80 \mathrm{mg}^{\text {day }}{ }^{-1}$ and a dose-dependency of the overall incidence of the duloxetine-associated AEs has been reported from a controlled dose-ranging study [13]. AEs leading to premature treatment termination were also less frequent in the present than in previous controlled SUI studies [13-19].
The comorbidities and comedications reported in the present study are typical for what is seen in this age group in Germany, as also reflected by observational studies with urge incontinence medication [27]. The prevalence of hypertension, heart disease, lipid metabolism disorders and diabetes indicates that SUI patients have a rather high cardiovascular risk. While duloxetine can have minor effects on blood pressure and/or heart rate, a comprehensive analysis based upon studies in SUI [20] or across all indications [34] indicates a lack of major cardiovascular AEs, a finding confirmed by the present observational study in SUI patients.

There is an ongoing discussion about the risk of suicidal ideation and suicide attempts associated with the use of SSRIs $[22,23]$ and some case reports have also described suicidal behaviour in patients receiving duloxetine in its psychiatric indication $[35,36]$. Based on a request by the European regulatory authorities, our study has specifically explored potential effects on suicidality, particularly within the group of patients treated by PCPs. In that group a surprisingly high depression score at baseline was found with $26.6 \%$ and $15.6 \%$ of SUI-treated patients being classified as a probable or most likely case of depressive disorder, respectively, prior to duloxetine exposure. Moreover, thoughts of self-harm, suicidal thinking and a history of suicide attempts were also frequent at baseline. However, despite reporting on a total of 8923 duloxetine-treated patients yielding a total exposure of 3233 patient-years, our study did not report suicidal attempts. However, the fact that we were unable to recruit the originally planned number of patients, may have somewhat limited our ability to detect suicidal attempts. Moreover, suicidal attempts may be under-reported, but our use of a reference group at least partly may have corrected for this. A previous metaanalysis of 702 controlled SSRI studies including 87650 patients had reported an odds ratio for suicide attempts as compared with placebo-treated patients of 2.28 and a number needed to harm of 634 [22]. While the present study clearly does not have the same technical strength as a meta-analysis of controlled studies, a lack of suicide attempts in our 8923 patients as well as in the 958 duloxetine patients in placebo-controlled SUI studies [20] is certainly encouraging. Whether these findings are also applicable to patients receiving duloxetine for psychiatric indications remains unclear as a recent analysis covering almost 100000 patients in placebo-controlled studies found that suicidal behaviour or ideation were extremely rare when antidepressants were used in non-psychiatric indications [23].

Finally, we have used the large database of our study to explore factors potentially associated with AE occurrence using logistic regression analysis. This analysis demonstrated in most models that reported AEs were related to physician specialization ( $P C P<U R O<G Y N$ ), starting dose $\left(20<40<80 \mathrm{mg} \mathrm{day}^{-1}\right)$, concomitant medication in general and concomitant overactive bladder medication in 
particular. Interestingly, patients reporting a higher depression questionnaire score at baseline were significantly less likely to report AEs during duloxetine treatment. Increasing age had a small but statistically significant effect on $A E$ occurrence, similar to what has been reported with urgency incontinence medications [27]. While the absolute numbers of AEs reported during OTHER treatment were lower than with duloxetine treatment, concomitant medication and specifically concomitant overactive bladder medications also were associated with a greater risk for reported AEs.

In conclusion, our observational study with a large number of patients and large overall duloxetine exposure in terms of patient years confirmed the qualitative pattern of AEs reported from controlled studies but with a much lower overall incidence. Moreover, we did not detect any rare, previously unreported AEs. Our study did not provide evidence for an increased suicidality risk for duloxetine treatment in SUI patients. In a more general vein, we propose that the use of a carefully chosen comparator group may strengthen the scientific value of observational studies.

\section{Competing Interests}

The statistical analysis was performed by the author D. Quail, an employee of Eli Lilly and Company. Authors A. Minarzyk, I. Schwerdtner and $\mathrm{H}$.-J. Weber are employees of Lilly Deutschland GmbH, authors M. C. Michel and H. D. Methfessel have been paid consultants for the design and analysis of this study. After completion of the study and its analysis M. C. Michel became an employee of Boehringer Ingelheim Pharma GmbH \& Co KG.

This manuscript is based upon a study funded and conducted by Lilly Deutschland GmbH. The study design and statistical analysis plan were jointly developed by the authors, who also jointly interpreted the data scientifically and prepared, reviewed and approved the final manuscript. Dr Martina Manning, an employee of Lilly Deutschland, also contributed substantially to the design, conduct and evaluation of the study.

\section{REFERENCES}

1 Abrams P, Cardozo L, Fall M, Griffiths D, Rosier P, Ulmsten U, van Kerrebroeck P, Victor A, Wein A. The standardisation of terminology of lower urinary tract function: report from the standardisation sub-committee of the International Continence Society. Neurourol Urodyn 2002; 21: 167-78.

2 Townsend MK, Curhan GC, Resnick NM, Grodstein F. The incidence of urinary incontinence across Asian, Black, and White women in the United States. Am J Obstet Gynecol 2010; 202: 378e1-e7.
3 Hampel C, Artibani W, Espuna Pons M, Haab F, Jackson S, Romero J, Gavart S, Papanicolaou S. Understanding the burden of stress urinary incontinence in Europe: a qualitative review of the literature. Eur Urol 2004; 46: 15-27.

4 Hunskaar S, Lose G, Sykes D, Voss S. The prevalence of urinary incontinence in women in four European countries. BJU Int 2004; 93: 324-30.

5 Coyne KS, Zhou Z, Thompson C, Versi E. The impact on health-related qualify of life of stress, urge and mixed urinary incontinence. BJU Int 2003; 92: 731-5.

6 Curie CJ, McEwan P, Poole CD, Odeyemi IAO, Datta SN, Morgan CL. The impact of overactive bladder on health-related utility and quality of life. BJU Int 2006; 97: 1267-72.

7 Schimpf MO, Patel M, O'Sullivan DM, Tulikangas PK. Difference in quality of life in women with urge urinary incontinence compared to women with stress urinary incontinence. Int Urogynecol J 2009; 20: 781-6.

8 Andersson K-E, Appell R, Cardozo L, Chapple CR, Drutz H, Finkbeiner A, Haab F, Vela Navarrete R. Pharmacological Treatment of Urinary Incontinence. Plymouth: Plymbridge Distributors Ltd, 1999; 447-86.

9 Katofiasc MA, Nissen J, Audia JE, Thor KB. Comparison of the effects of serotonin selective, norepinephrine selective, and dual serotonin and norepinephrine reuptake inhibitors on lower urinary tract function in cats. Life Sci 2002; 71: 1227-36.

10 Thor KB, Katofiasc MA. Effects of duloxetine, a combined serotonin and norepinephrine reuptake inhibitor, on central neural control of lower urinary tract function in the chloralose-anesthetized female cat. J Pharmacol Exp Ther 1995; 274: 1014-24.

11 Boy S, Wirth B, Knapp P, Braun PM, Haferkamp A, Schurch B. Facilitatory neuromodulative effect of duloxetine on pudendal motor neurons controlling the urethral pressure: a functional urodynamic study in healthy women. Eur Urol 2006; 50: 119-25.

12 Michel MC, Oelke M. Duloxetine in the treatment of stress urinary incontinence. Women's Health 2005; 1:345-58.

13 Norton PA, Zinner NR, Yalcin I, Bump RC. Duloxetine versus placebo in the treatment of stress urinary incontinence. Am J Obstet Gynecol 2002; 187: 40-8.

14 Dmochowski RR, Miklos JR, Norton PA, Zinner NR, Yalcin I, Bump RC. Duloxetine versus placebo for the treatment of North American women with stress urinary incontinence. J Urol 2003; 170: 1259-63.

15 Cardozo L, Drutz HP, Baygani S, Bump RC. Pharmacological treatment of women awaiting surgery for stress urinary incontinence. Am J Obstet Gynecol 2004; 104: 511-9.

16 Millard RJ, Moore K, Rencken R, Yalcin I, Bump RC. Duloxetine vs placebo in the treatment of stress urinary incontinence: a four-continent randomized clinical trial. BJU Int 2004; 93 : 311-8.

17 van Kerrebroeck P, Abrams P, Lange R, Slack M, Wyndaele JJ, Yalcin I, Bump RC. Duloxetine versus placebo in the 
treatment of European and Canadian women with stress urinary incontinence. BJOG 2004; 111:249-57.

18 Bump RC, Norton PA, Zinner NR, Yalcin I. Mixed urinary incontinence symptoms: urodynamic findings, incontinence severity, and treatment response. Obstet Gynecol 2003; 102: 76-83.

19 Ghoniem GM, Schagen van Leeuwen J, Elser DM, Freeman RM, Zhao YD, Yalcin I, Bump RC. A randomized controlled trial of duloxetine alone, pelvic floor muscle training alone, combined treatment and no active treatment in women with stress urinary incontinence. J Urol 2005; 173: 1647-53.

20 Hurley DJ, Turner CL, Yalcin I, Viktrup L, Baygani SK. Duloxetine for the treatment of stress urinary incontinence in women: an integrated analysis of safety. Eur J Obstet Gynecol 2006; 125: 120-8.

21 Brunton S, Wang F, Edwards SB, Crucitti AS, Ossanna MJ, Walker DJ, Robinson MJ. Profile of adverse events with duloxetine treatment. A pooled analysis of placebo-controlled studies. Drug Saf 2010; 33: 393-407.

22 Fergusson D, Doucette S, Glass KC, Shapiro S, Healy D, Hebert $P$, Hutton B. Association between suicide attempts and selective serotonin reuptake inhibitors: a systematic review of randomised controlled trials. Br Med J 2005; 330: 396.

23 Stone $M$, Laughren $T$, Jones ML, Levenson M, Holland PC, Hughes A, Hammad TA, Temple R, Rocherster G. Risk of suicidality in clinical trials of antidepressants in adults: analysis of proprietary data submitted to the US Food and Drug Administration. Br Med J 2009; 339: b2880.

24 Manning M, Gotsch U, Minarzyk A, Quail D, Gross A, Pages I, Methfessel HD, Michel MC. How are women with SUl-symptoms treated with duloxetine in real life practice? Prelimnary results from a large observational study in Germany. Int J Clin Pract 2009; 63: 1724-33.

25 Grulke N, Bailer H, Blaser G, Geyer M, Hinz A, Schmutzer G, Brähler E, Albani C. Depressivitäts-Screening - zwei Fragen für die Praxis. Wien Med Wochenschr 2005; 155: 297-302.

26 Albani C, Blaser G, Völker J, Geyer M, Schmutzer G, Bailer H, Grulke N, Brähler E, Traue HC. Ambivalence over emotional expressiveness: psychometric evaluation of the AEQ-G18 in a representative German survey. Psychosoc Med 2007; 4: Doc10.

27 Michel MC, Wetterauer U, Vogel M, de la Rosette JJMCH. Cardiovascular safety and overall tolerability of solifenacin in routine clinical use. Drug Saf 2008; 31: 505-14.

28 Schneider T, Marschall-Kehrel D, Hanisch J-U, Michel MC. Do gender, age or life style factors affect responses to anti-muscarinic treatment in overactive bladder patients? Int J Clin Pract 2010; 64: 1287-93.

29 Khan A, Schwartz K. Suicide risk and symptom reduction in patients assigned to placebo in duloxetine and escitalopram clinical trials: analysis of the FDA summary basis of approval reports. Ann Clin Psychiatry 2012; 19: 31-6.

30 Castro-Diaz D, Palma PCR, Bouchard C, Haab F, Zepeda Contreras S, Ridorigues Ginorio H, Voss S, Yalcin I, Bump RC. Effect of dose escalation on the tolerability and efficacy of duloxetine in the treatment of women with stress urinary incontinence. Int Urogynecol J 2007; 18: 919-29.

31 Michel MC, Schneider T, Krege S, Goepel M. Do gender or age affect the efficacy and safety of tolterodine? J Urol 2002; 168: 1027-31.

32 Michel MC, de la Rosette JJMCH, Piro M, Schneider T. Comparison of symptom severity and treatment response in patients with incontinent and continent overactive bladder. Eur Urol 2005; 48: 110-5.

33 Witte LPW, Peschers U, Vogel M, de la Rosette JJMCH, Michel $M C$. Does the number of previous vaginal deliveries affect overactive bladder symptoms or their response to treatment? LUTS 2009; 1:82-7.

34 Wernicke J, Lledo A, Raskin J, Kajdasz DK, Wang F. An evaluation of the cardiovascular safety profile of duloxetine. Findings from 42 placebo-controlled studies. Drug Saf 2007; 30: 437-55.

35 Reeves RR, Brister JC. Serious suicide attempt with duloxetine treatment. South Med J 2008; 101: 769.

36 Salem BA, Karam EG. Duloxetine and suicide attempts: a possible relation. Clin Pract Epidemiol Ment Health 2008; 4: 18. 